\title{
Gymnastikhøjskolen i Ollerup, das vivências iniciais a grande performance: um relato de experiência
}

Gymnastikhøjskolen i Ollerup, from initial experiences to the great performance: an experience report

Gymnastikhøjskolen i Ollerup, de las experiencias iniciales al alto rendimiento: un informe de experiencia

Ana Paula Dias de Souza ${ }^{\mathrm{I}}$, Andrize Ramires Costa ${ }^{\mathrm{II}}$

\begin{abstract}
Resumo
Este estudo teve como objetivo descrever a vivência em um programa de mobilidade acadêmica viabilizado pela International Sport and Culture Association (ISCA). Trata-se de um relato de experiência na escola dinamarquesa de ginástica Gymnastikhøjskolen i Ollerup, em Ollerup, Dinamarca. O intercâmbio teve duração de seis meses no primeiro semestre de 2018, cuja programação incluiu a prática e a realização de performances de ginástica. Dentre aspectos do ensino holístico da escola e a importância da internacionalização para o método ginástico Dinamarquês de Niels Bukh, a vivência agregou na minha formação pessoal e profissional.
\end{abstract}

Palavras-chave: Ginástica; Intercâmbio Educacional Internacional; Dinamarca

\begin{abstract}
The aim of this study was to describe the experience of an internship program made possible by International Sport and Culture Association (ISCA). This is an experience report at the Danish gymnastic school Gymnastikhøjskoleni Ollerup, in Ollerup, Denmark. The exchange lasted six months in the first half of 2018, and course included the practice and performance of gymnastics. Among aspects of the school's holistic teaching and the importance of internationalization for the Danish gymnastic method of Niels Bukh, the experience added to my personal and professional education.
\end{abstract}

Keywords: Gymnastics; International Educational Exchange; Denmark

\footnotetext{
${ }^{\text {I } U n i v e r s i d a d e ~ F e d e r a l ~ d e ~ P e l o t a s ~-~ U F P E L ~-~ E n d e r e c ̧ o: ~ R . ~ G o m e s ~ C a r n e i r o, ~} 1$ - Centro, Pelotas - RS, 96010-610 - e-mail: anadiasbueno@ gmail.com

${ }^{\text {II }}$ Universidade Federal de Pelotas - UFPEL - e-mail: andrize.costa@gmail.com
} 


\section{Resumen}

Este estudio tuvo como objetivo describir la experiencia en un programa de movilidad académica hecho posible por International Sport and Culture Association (ISCA). Este es un relato de experiencia en la escuela de gimnasia danesa Gymnastikhøjskolen i Ollerup, em Ollerup, Dinamarca. El intercambio duró seis meses en la primera mitad de 2018, cuyo cronograma incluyó la práctica y realización de actuaciones gimnásticas. Entre los aspectos de la educación holística de la escuela y la importancia de la internacionalización para la gimnasia danesa de Niels Bukh, la experiencia se sumó a mi entrenamiento personal y profesional.

Palabras clave: Gimnasia; Intercambio Educativo Internacional; Dinamarca

\section{Introdução}

Durante o período entre as guerras, a Ginástica Dinamarquesa foi desenvolvida e idealizada por Niels Bukh, que, em 1920, fundou na Dinamarca a Gymnastikhøjskolen i Ollerup, a primeira escola de ginástica do país pautada no estilo holístico de ensino, conhecido também como Folk High School (LANGLADE; LANGLADE, 1970). Esse estilo de ensino, muito popular na Dinamarca, utiliza um sistema não formal, no qual os alunos têm a oportunidade de explorar diversos tópicos sem se preocuparem com avaliações (BAGLEY; RUST, 2009).

O método desenvolvido por Bukh partiu de seus questionamentos a respeito do método sueco de Ling, o qual passou a incorporar mais vigor nos movimentos rítmicos que fluíam de forma interligada no intuito de desenvolver flexibilidade, mobilidade e liberdade (LANGLADE; LANGLADE, 1970). Além de mobilizar jovens do país, Bukh popularizou o método por meio de suas relações políticas, performances de ginástica em tours pelo mundo e propagandas (BONDE, 2009).

Atualmente, a Ginástica na Dinamarca é entendida como elemento chave para o desenvolvimento dos indivíduos em uma perspectiva lúdica, cultural, de aprendizagem e de relacionamentos (LACERDA, 2010). A divulgação e a prática de várias manifestações gímnicas pode ser evidenciada nos grandes eventos, como o Landstaevne ${ }^{1}$, que reúne milhares de pessoas e fomenta o esporte e a Ginástica para Todos (PATRÍCIO; BOROLETO, 2015). A Escola de Ginástica de Ollerup demarca essa característica rendendo-se ao prazer pela prática (TRUZZI, 1999).

No Brasil, a partir da década de 1990, professores e grupos iniciam um processo de internacionalização importante com a então Ginástica Geral proporcionada pela participação em eventos internacionais, com destaque para as Gymnaestradas Mundiais (SANTOS; SANTOS, 1999) e as bolsas de estudos viabilizadas com a filiação da Universidade Estadual de Campinas (Unicamp) à International

${ }^{1}$ Festival internacional realizado a cada quatro anos há 148 anos pela Associação Dinamarquesa de Esportes e Tiro. 
Sports and Culture Association(ISCA), que posteriormente foram ampliadas a outras instituições (AYOUB; BORTOLETO, 2013).

Esse processo de internacionalização foi muito importante para o fortalecimento de parcerias e para a ampliação da Ginástica Geral no Brasil: uma Ginástica sem restrições e regras e que, portanto, cria espaço para a diversidade e para a criatividade, em especial uma ginástica do prazer, da felicidade e do divertimento - diferente das ginásticas desportivas até então conhecidas (AYOUB, 2007).

Welch e Luostarinen (1998) refletem a respeito do conceito de internacionalização como o processo de crescente envolvimento em operações internacionais, conectando ambos os lados do processo. Diante disso, compreendendo a influência que a Europa ainda exerce na Ginástica brasileira, este estudo objetivou descrever a vivência em um programa de mobilidade acadêmica, especificando-se em: apresentar o ensino; descrever a montagem e as apresentações de ginástica; bem como discutir aspectos que envolvem a criação de performances de Ginástica inserido do contexto da Gymnastikhøjskolen i Ollerup.

\section{Metodologia}

Para o presente estudo, optou-se por um relato de experiência como método de pesquisa, com caráter descritivo-reflexivo. Dessa forma, o relato baseou-se na minha experiência/vivência como aluna na Gymnastikhøjskolen i Ollerup no primeiro semestre de 2018 e participante das performances de ginástica do respectivo semestre, juntamente com registros feitos nesse período, como vídeos, fotos e conteúdos disponíveis em grupos de redes sociais.

Para fundamentar o estudo, também utilizamos a técnica de documentação indireta descrita por Lakatos e Marconi (1991), a qual possibilita reunir informações prévias sobre o assunto de interesse utilizando pesquisa bibliográfica, o que permite selecionar, fichar e arquivar tópicos de interesse para a pesquisa, juntamente com informações e dados pesquisados em outras produções, como livros, sites, jornais e outros. 


\section{Resultados e discussão}

\section{$3.1 \mathrm{O}$ ensino}

Embora o intercâmbio tenha acorrido durante a graduação, a mobilidade oferecida pelo programa International Youth Leader Education (IYLE), viabilizado pela ISCA, trata-se de "ensino superior para ensino não formal". Ao descrever as diferentes formas de ensino, Bianconi e Caruso (2004) elencam três classificações: a educação formal, presente no ensino institucionalizado, cronologicamente gradual e hierarquicamente estruturado; a informal, que está relacionado ao acúmulo de conhecimentos através experiência diária em casa, no trabalho e no lazer; e a educação não formal, como qualquer tentativa educacional organizada e sistematizada fora dos quadros do sistema formal de ensino. Considerando que a atuação profissional na área de esportes é influenciada pela formação inicial, não formal e informal (CARBINATTO et al., 2017), podemos sugerir que a mobilidade acadêmica proporcionada pela IYLE assume um papel complementar na formação dos participantes.

O ensino não formal oferecido pela escola de ginástica de Ollerup segue um modelo muito popular na Dinamarca que foi desenvolvido pelo dinamarquês Nikolaj Frederik Severin Grundtvig, em 1830.As escolas são popularmente conhecidas como Folk High School ou em dinamarquês Folkehøjskole que, pela tradução da palavra, significa escola de pessoas. Os alunos também são livres para escolher os tópicos de interesse sem preocuparem-se com notas (THE ASSOCIATION OF FOLK HIGH SCHOOL IN DENMARK, 2019).

Embora a escola de Ginástica de Ollerup não exigisse um perfil específico além da idade entre 18 e 30 anos e proficiência em inglês, ocorreu uma pré-seleção na universidade a qual eu estava vinculada devido à instituição poder ocupar apenas duas vagas no programa da IYLE, no primeiro semestre de 2018. Dessa forma, se fez relevante minha trajetória na ginástica e universidade para ocupar a vaga.

Os tópicos eram variados, com atividades comuns a todos os alunos e aulas específicas de livre escolha, como as opções trimestrais e as linhas de especialização para todo o semestre. As linhas eram na maior parte atividades práticas, sendo elas Parkour, Dança e modalidades gímnicas. Independente da linha, todos os alunos tinham a oportunidade de praticar muita ginástica através das aulas comuns e as performances, além das semanas especiais como a Old Week (semana antiga), com aulas de ginástica nos moldes dos tempos de NeilsBukh. Além do mais, fora dos horários das aulas, os espaços das mais diferentes linhas ficavam disponíveis para os alunos. 
Alguns tópicos são direcionados apenas para alunos internacionais, como Organizações não governamentais, Idiomas e Estudos Interculturais (OLLERUP INTAC, 2020). Ao final do semestre, além do diploma da escola, os alunos internacionais recebem um certificado da ISCA constando todas as atividades específicas para os intercambistas.

Referente às aulas, é importante mencionar a liberdade do professor para desenvolver as atividades nesse estilo de ensino (THE ASSOCIATION OF FOLK HIGH SCHOOL IN DENMARK, 2019). Dentro de suas linhas e aspectos básicos da Folk High School, como o diálogo e igualdade entre professores e alunos, o professor é livre para escolher os caminhos metodológicos e aproveitar suas potencialidades.

Embora seja notório o menor aprofundamento teórico, se comparado ao ensino formal, principalmente pelas atividades das linhas que abrangem em sua maior parte a prática como mencionado anteriormente, o significado de se mover dentro escola de ginástica de Ollerup contribuiu significativamente para reflexões a respeito das possibilidades proporcionadas pelo esporte. Nesse aspecto, Kunz (2004), ao refletir sobre a "transformação didático-pedagógica" no esporte, aponta que a “perfeição" não está nos padrões da modalidade em si, mas sim a concretização pelo prazer e satisfação do aluno.

Para complementar a apropriação dos conteúdos, tive preocupação em registar o máximo possível, com vídeos, fotos e anotações. Minha experiência prévia também contribuiu para reflexão crítica sobre as atividades, pois em algumas ocasiões na rotina da escola, os professores contavam histórias referentes à guerra, à Ginástica Dinamarquesa, à vida de Neils Bukh e a outros assuntos.

\subsection{A grande performance da turma F18}

A Grande Performance, nomeada também de Gym Gala, trata-se de uma apresentação de ginástica incluída na programação da escola de Ollerup no primeiro semestre de 2018. Nela, cerca de 220 alunos foram participantes.

A Gym Gala, pelo seu quantitativo de integrantes, o caráter demonstrativo com as diversas sequências de Ginástica Dinamarquesa, dialogando com outras manifestações gímnicas praticadas na escola, teve características peculiares desde o processo de montagem ao resultado final. Essas características vão ao encontro com a Ginástica de Grande Área (GGA), que segundo (BUENO, 2004), “é uma ginástica de demonstração, realizada em grandes superfícies, utilizando materiais de pequeno e grande porte e envolvendo um elevado número de participantes". A autora ainda ressalta que a GGA foi 
um importante veículo de demonstração utilizado pelas Escolas de Ginástica europeias, pelo seu potencial de disseminar ideias dos métodos ginásticos desenvolvidos, contando ainda com apoio político. Embora o número de participantes para GGA seja estabelecido como "elevado" na literatura, o manual Ginástica Para Todos da Federação Internacional de Ginástica (2019), referente a performances de grandes grupos, estabelece o quantitativo mínimo de 200 pessoas em uma apresentação com o qual podemos ter base ao descrever a Gym Gala com o caráter de GGA.

A programação principal para a apresentação do Gym Gala incluía turnê internacional em cidades da Alemanha, como Weinheim ${ }^{2}$ e Heindelberg ${ }^{3}$,bem como apresentações internas na escola em Ollerup, Dinamarca. Algumas apresentações na Alemanha ocorreram na semana do Landesturnfest, um grande evento de ginástica com competições e festivais em diversos locais da cidade de Weinheim. Outro evento que contemplou apenas um fragmento da performance foi o VšesokolskýSlet em Praga, República Tcheca, que é um grande evento realizado pela associação tcheca de Educação Física - SOKOL - e traz forte tradição de GGA (BUENO, 2004).

\subsection{Os ensaios}

Os ensaios iniciaram na metade do semestre com encontros fixos e cerca de quatro horas de duração. O horário utilizado era destinado para atividades comuns a todos os alunos da escola, chamado em dinamarquês de fallesskab. Essa palavra, sem tradução específica para o português, insere conceitos bem peculiares da cultura dinamarquesa que, como destacado por Cristiansen (2015), se trata da compreensão a respeito de comunidade, o que incorpora valores conectados ao sentimento pessoal de pertencimento, o qual é naturalizado como um fato visual e cultural para os dinamarqueses. Desse modo, os ensaios da performance evidenciaram valores da cultura dinamarquesa, idealizado por um trabalho conjunto para o bem comum.

A princípio, a performance não foi estruturada de forma linear, ou seja, do início para o fim. Primeiramente aprendemos as sequências coreografadas, para depois entender a formação espacial e por fim as entradas e saídas com a formação geral da performance. As etapas eram explicadas e mostradas em forma de mapa ou fluxograma, no qual os participantes visualizavam previamente sua colocação. Esse tipo de composição, como ressalta Bueno (2004) ao descrever sobre GGA, trabalha com diferentes formações e deslocamento, o qual exige muito planejamento.

${ }^{2}$ Weihem é uma cidade da Alemanha, localizada ao Noroeste do estado de Baden-Württemberg no sudoeste da Alemanha.

${ }^{3}$ Heidelberg, cidade localizada às margens do rio Neckar, no sudoeste da Alemanha. 
O aprendizado das sequências ocorria nas quadras e em espaços externos amplos e gramados. Os professores faziam jogos para aquecimento, e interação entre os participantes e posteriormente o passo a passo das sequências. Em seguida éramos divididos em grupos para ajuda mútua no aprendizado das sequências. Ao final dos ensaios, assistíamos uns aos outros como forma de apreciação do trabalho e aprendizagem. Segundo Souza (1997), essa etapa na criação de uma performance na GPT é definida como fase de demonstração, o qual reforça o trabalho em grupo, a sensação de pertencimento e também o reconhecimento de seus pares.

Além disso, os vídeos das sequências eram compartilhados para que pudéssemos ensaiar em outros horários. Ao final da montagem, os ensaios seguintes eram destinados ao aperfeiçoamento, como correções das posições, memorização das sequências e os sentimentos expressados nas etapas. Também havia grande incentivo por parte dos professores para que os alunos se ajudassem e que todos aproveitassem o processo. Sempre repetiam a seguinte frase: "Tudo que importa é se divertir, aproveitar o momento".

Figura 1-Mapa com posições da cena Opening. De um lado Piger (meninas) do outro drenge (meninos)

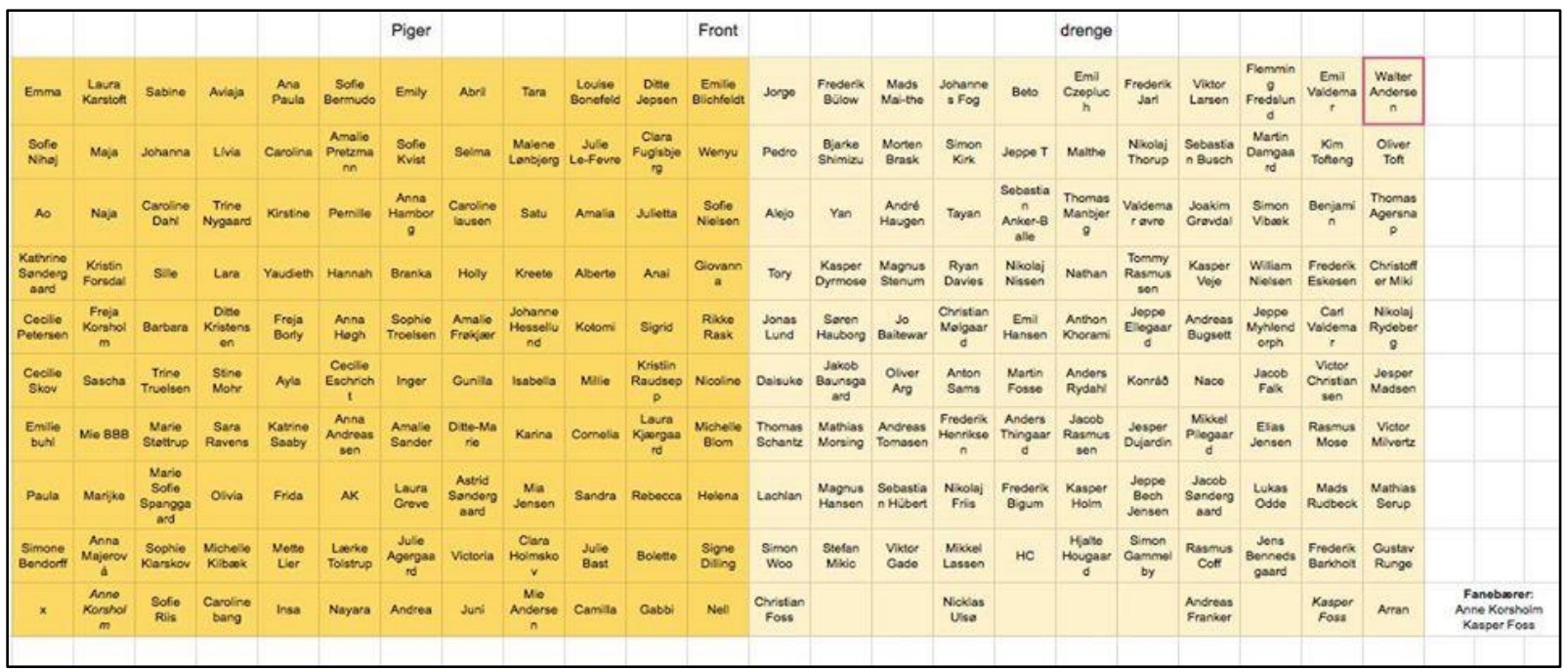

Fonte: Arquivo pessoal ${ }^{4}$

\subsection{O processo de composição coreográfica do Gym Gala}

O processo de composição coreográfica refere-se ao conteúdo a ser contextualizado nas diversas variáveis, como movimentos, músicas, vestimentas e outros. Para Marcassa (2004), a temática em uma

${ }^{4}$ Printscreen editado pelos autores de uma planilha com o mapa das posições dos alunos na coreografia. 
coreografia de ginástica atribui sentido ao conteúdo por meio de combinar, sequenciar e contextualizar ações, gestos e posturas em um "texto". Na Gym Gala, a temática foi dividida em 10 cenas principais, as quais valorizavam Ginástica Dinamarquesa, a escola de Ollerup e a cultura expressas pelas músicas tradicionais, as práticas gímnicas nos tempos de Niels Bukh e aspectos contemporâneos com as modalidades da escola e os equipamentos modernos como o airtrack ${ }^{5}$.

Embora a palavra "cultura" tenha grande abrangência no seu sentido, ela pode exprimir determinadas dimensões da vida social (ORTIZ, 2010, p. 20). No caso desse relato, essas dimensões estão aliadas a um contexto de ensino para um público jovem e aspectos tradicionais da Ginástica Dinamarquesa de uma escola fundada em 1920. Embora as transformações ao longo das gerações, é possível perceber a apreciação histórica passada entre as gerações e através do ensino da ginástica. "Certamente, é na esfera cultural que os símbolos são gestados e preenchidos de sentido; mas, eles se transformam com o peso da história" (ORTIZ, 2010,p. 20).

Figura 2- Cartaz no mural com a ordem das cenas da performance

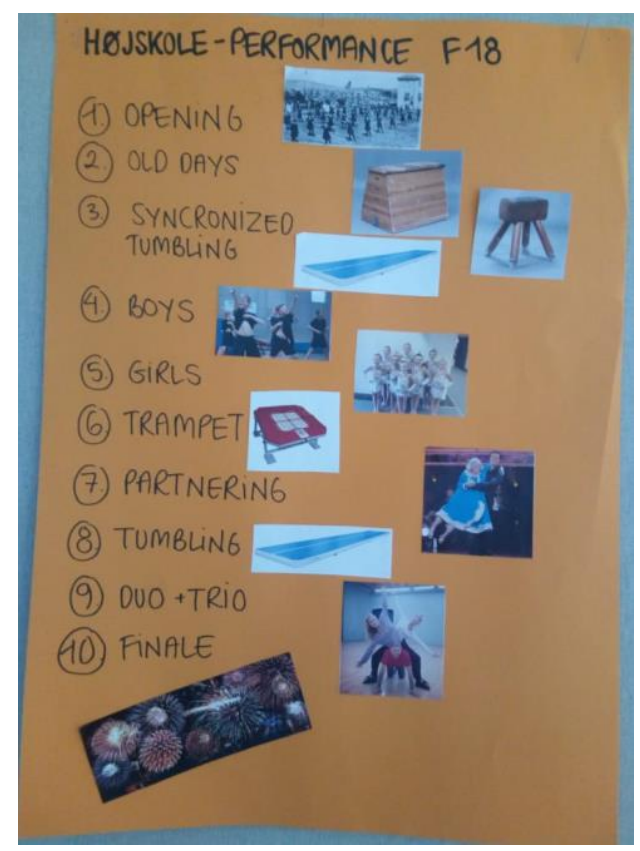

Fonte: Captura feita pelos autores ${ }^{6}$

\subsection{A Tradição pelas músicas}

Se tratando de uma performance de grande área, deve existir harmonia entre movimento, formação e música (BUENO, 2004). A trilha utilizada na Gym Gala foi composta, em sua maioria, por

${ }^{5}$ Colchão inflável com material e proporções específicas para execução de acrobacias de ginástica.

${ }^{6} \mathrm{O}$ cartaz exposto no mural da escola Gymnastikhøjskolen i Ollerupdurante o período de ensaios da Gym Gala. 
músicas tradicionais de uma Folk High School. Os alunos estavam bem familiarizados com as músicas, pois eram cantadas frequentemente nos encontros matinais chamados de morningassembly. Como destaca Eichberg (2006), o canto é uma forte característica das práticas de ensino experimentadas em uma Folk High School.

Esse costume influenciou na tradição do canto em alguns eventos esportivos no país, principalmente em eventos de ginástica, uma vez que o esporte e a Folk High Schoolestão atrelados historicamente (EICHBERG, 2006).

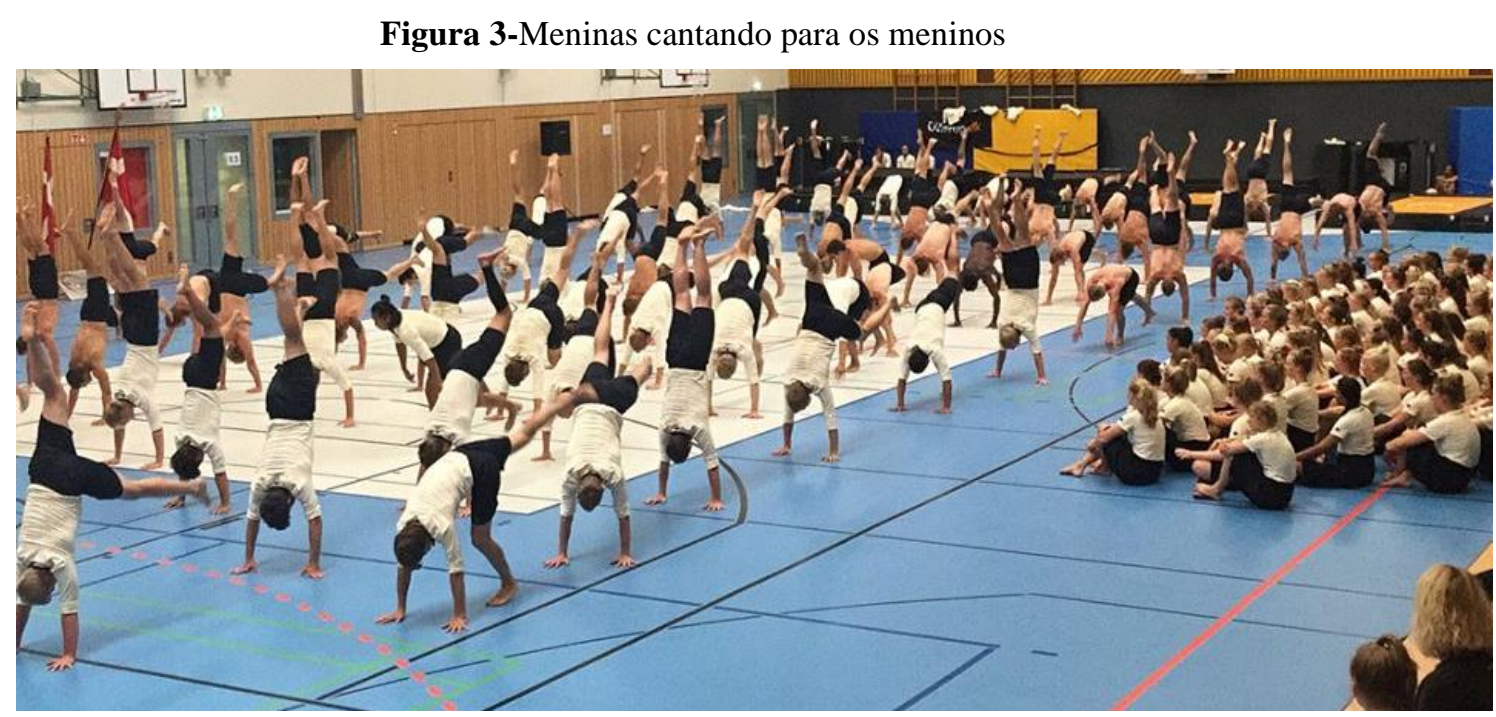

Fonte: Arquivo pessoal ${ }^{7}$

\subsection{A grande Performance}

A primeira apresentação foi na escola de Ollerup, área externa destinada à comunidade local e aos convidados dos alunos. O espaço onde ocorriam os ensaios gerais sediou outras apresentações de eventos da escola. O resultado da primeira apresentação gerou muita euforia entre os alunos.

Na apresentação principal do Landesturnfest, o quantitativo de alunos e o tipo de performance não comportavam os limites de tempo e espaço. Dessa forma, a performance foi fragmentada em duas coreografias: uma somente com as meninas, que trouxe fortes características da Ginástica Dinamarquesa;bem como o Tumbling, com sequências de saltos no mini-trampolim e airtrack. Eram feitos rodízios entre os dias do evento para que todos tivessem a oportunidade de se apresentar.

\footnotetext{
${ }^{7}$ Imagem do acervo pessoal dos autores, com registros compartilhados entre professores e alunos durante todo o semestre.
} 
Para VšesokolskýSlet, o convite para a participação surgiu depois que o semestre estava em andamento, direcionando-se apenas para as meninas, pois os meninos estariam em um outro programa exclusivo da escola nas datas do evento. O grupo se apresentou durante uma semana em diversos locais na cidade de Praga, República Tcheca, e também na cerimônia principal do VšesokolskýSlet, em uma grande arena que comportava milhares de pessoas. A coreografia contemplou um fragmento da Gym Gala com a sequência das meninas.

\section{Conclusões}

Viver inserida em um contexto tradicional dinamarquês e o modo que eles praticam e entendem a ginástica, me trouxe uma nova compreensão a respeito dessa prática. Pois ao estudar ao longo da minha graduação seu processo histórico social e depois vivenciar um método em sua essência, foi sem dúvida um marco na minha formação profissional e pessoal.

Considerando o processo de internacionalização com trocas entre dois organismos, pude agregar às minhas experiências do intercâmbio em projetos no Brasil que abrangem ensino pesquisa e extensão. Não se trata da reprodução de conhecimentos de outro país, mas sim reunir à minha trajetória para aplicar as potencialidades dos conhecimentos adquiridos visando nosso contexto. No aspecto de mercado, sem dúvidas se tratou de um diferencial, bem como a proficiência em idiomas.

Participar dos grandes eventos de ginástica, como o Landesturnfest na Alemanha, e o V̌̌esokolskýSlet na República Tcheca, foram grandes oportunidades para apreciar diferentes manifestações gímnicas, que reuniram milhares de pessoas de diversos países. Assistir a uma performance em massa, com mais de mil pessoas, como na V̌sesokolskýSlet, me levou a diversos questionamentos a respeito do processo que mobilizou tantos indivíduos em uma manifestação que envolve esporte, cultura e história.

Participar da performance, desde os processos iniciais até o produto final, contribuiu para reflexões no trato com a ginástica, estabelecendo uma nova relação com o "resultado". Nesse caso, equiparando a ginástica ao contexto brasileiro, falar de resultado se refere muitas vezes a conceitos do alto rendimento. Vislumbrar um produto como a satisfação dos praticantes, o aprendizado, o trabalho conjunto, trouxe uma significância para as potencialidades da ginástica, que agregou na minha formação. 


\section{Referências}

AYOUB, Eliana. Ginástica geral e Educação Física escolar. 2. ed. Campinas: Ed. da Unicamp, 2007

AYOUB, Eliana; BORTOLETO, Marco Antônio C. Uma vida de dedicação à Ginástica: inspirando gerações. Conexões, v. 11, n. 3, p. 202-207, 2013.

BAGLEY, Sylvia S.; RUST, Val D. Community-based folk high schools in Norway, Sweden, and Denmark. In: Community college models. Springer, Dordrecht, 2009. p. 279-298.

BIANCONI, M. Lucia; CARUSO, Francisco. Educação não-formal. Ciência e cultura, v. 57, n. 4, p. 2020, 2005.

BUENO, Thaís Franco. Ginástica de grande área: uma realidade possível no contexto escolar. 2004. 130f. Dissertação (Mestrado em Educação Física), Faculdade de Educação Física, Universidade Estadual de Campinas, Campinas, 2004.

CARBINATTO, Michele Viviane et al. Conhecimentos de Acadêmicos de Educação Física sobre a Ginástica a partir da Percepção de Docentes do Ensino Superior. Revista de Graduação USP, v. 2, n. 3, 2017.

CHRISTIANSEN, Lene Bull. 'Africa is a national cause': Race and nation in development aid communication--A Danish case study. Critical Race \& Whiteness Studies, v. 11, n. 1, 2015.

EICHBERG, Henning. Song and movement: Danish experiences and educational challenges for Sport for all. In: Movement Recreation for All. Central Bookstore of Physical Culture, 2006. p. 47-60. Disponível em: http://citeseerx.ist.psu.edu/viewdoc/download?doi=10.1.1.573.2772\&rep=rep1\&type=pdf Acesso em: 23 mar. 2020.

FÉDÉRATION INTERNATIONALE DE GYMNASTIQUE (FIG). Gymnastics For All Manual. 2019. Disponível em: https://www.gymnastics.sport/publicdir/rules/files/en_Gymnastics\%20for\%20All\%20Manual,\%20Editio n\%202019.pdf Acesso em: 19 fev. 2020.

ISCA. News. IYLE Turned15. 2010. Disponível em: http://isca-web.org/english/news/iyleturned15/ Acesso em: 30 mar. 2020.

KUNZ, Elenor. Transformação Didático-Pedagógica. Ijuí, RS: Editora Unijuí, 2004.

LACERDA, Débora Jucá. A influência dos materiais, da estrutura e do espaço na prática da Ginástica de acrobacias. In: Fórum Internacional de Ginástica Geral, 5., 2010, Campinas /SP. Anais, editora da Unicamp, 2010. p.290-293.

LAKATOS, Eva. M., MARCONI, M. A. Fundamentos de metodologia científica. 3. ed. São Paulo: Atlas, 1991.

LANDESTURNFEST.

Übersichtsplan.

Disponível

em:

https://www.landesturnfest.de/mitmachen/turnfestzentrum/uebersichtsplan/ Acesso em: 12 out. 2019. 
LANGLADE, Alberto; LANGLADE, Nelly. R.; Teoria General de La Gimnasia. Buenos Aires, Argentina: Editorial Stadium, 1970.

MARCASSA, Luciana Pedrosa. Metodologia do ensino da ginástica: novos olhares, novas perspectivas. Pensar a Prática, v. 7, n. 2, p. 171-186, 2004.

OLLERUP INTAC. Tomorrow's Sport Leaders: Academy of Physical Education Ollerup. Dinamarca:

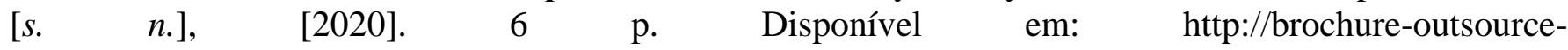
dk.com/ollerup_intac/intro/mobile/index.html\#p=1 Acesso em: 11 fev. 2020.

ORTIZ, Renato. A problemática cultural no mundo contemporâneo. Política \& Sociedade, v. 16, n. 35, p. 17-66, 2017.

PATRÍCIO, Tamiris Lima; BORTOLETO, Marco Antonio Coelho. Festivais ginásticos: Princípios formativos na visão de especialistas. Conexões, v. 13, p. 98-114, 2015.

BONDE, Hans. Nationalism in the age of extremes: taking danish gymnastics to the world. The International Journal of the History of Sport, v. 26, n. 10, p. 1414-1435, 2009.

SANTOS, José Carlos Eustáquio; SANTOS, NGM dos. História da ginástica geral no Brasil. Jundiaí: Fontoura, 1999.

SOUZA, Elizabeth P. M. Ginástica Geral: uma área do conhecimento da Educação Física. 1997.Tese (Doutorado) - Faculdade de Educação Física, Universidade Estadual de Campinas.

THE ASSOCIATION OF FOLK HIGH SCOOL IN DENMARK (FFD). Danish Folk High School: Højskolerne. Copenhague: $\quad$ Dinamarca, 2019.2 Disponível em: https://www.danishfolkhighschools.com/media/23511/19-danishfolkhighschool-haefte-web.pdfAcesso em: 16 fev. 2020.

TRUZZI, Luciano. Gymnastikhøjskolen i Ollerup: Uma experiência. 1999. 85f. Trabalho de Conclusão de Curso (Graduação) - Universidade Estadual de Campinas, São Paulo, 1999.

WELCH, Lawrence S.; LUOSTARINEN, Reijo. Internationalization: Evolution of a concept. Journal of general management, v. 14, n. 2, p. 34-55, 1988.

\section{Como citar este artigo}

SOUZA, A. P. D.; COSTA, A. R. Gymnastikhøjskolen i Ollerup, das vivências iniciais a grande performance: um relato de experiência. Revista Kinesis, Santa Maria, v.39, p.01-12, 2021.

* O presente trabalho não contou com apoio financeiro de nenhuma natureza para sua realização. 